\title{
Decreased Proliferation and Abnormal Differentiation of Human Mesenchymal Stromal Cells in Steroid-Induced Osteonecrosis of Femoral Head
}

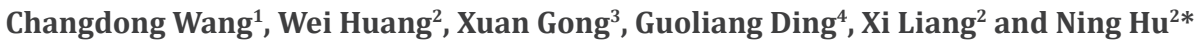 \\ ${ }^{1}$ Department of Biochemistry and Molecular Biology, Molecular Medicine and Cancer Research Center, Chongqing Medical University, Chongqing 400016, China \\ ${ }^{2}$ Department of Orthopaedic Surgery, the First Affiliated Hospital of Chongqing Medical University, Chongqing 400016, China \\ ${ }^{3}$ Department of outpatient, Chongqing Zhongshan Hospital, Chongqing, 400013, China \\ ${ }^{4}$ Department of Orthopaedic Surgery, the Second Affiliated Hospital of Baotou Medical College of Inner Mongolia University of Science and Technology,
} Baotou, 014030, China

Received: July 09, 2014; Accepted: July 28, 2014; Published: August 14, 2014

*Corresponding author: Ning Hu, Department of Orthopedic Surgery, Chongqing Medical University, Chongqing, China, Tel: 8618-6967-61022; Fax: 8623-8901-1212; E-mail: 1276321387@qq.com

\begin{abstract}
Objectives: To investigate if the pathogenesis of steroid-induced osteonecrosis of femoral head (ONFH) is associated with abnormal proliferation and differentiation of human mesenchymal stromal cells (MSCs) at the proximal femur.

Methods: Using isolated human MSCs and sections of whole femoral heads, we analyzed the proliferative capacity and osteogenic, angiogenic and adipogenic differentiation of human MSCs at the proximal femur.

Results: The proliferation of MSCs from patients with corticosteroid-induced $\mathrm{ONFH}$ is decreased. The down-regulated expression of BMP2, BMP7, BMP9 and Osteopontin provides supportive evidence corticosteroid-induced inhibition of osteogenesis. Down-regulation of HIF1 $\alpha$, VEGF and VWF by glucocorticoids is directly responsible for decreased angiogenesis. Over-expression of PPAR 2 and (442)aP2 suggests that the corticosteroids may induce MSCs to differentiate into adipocytes.

Conclusions: Our findings suggest steroids may reduce the proliferative activity of MSCs, down-regulate the expression of osteoblast differentiation factors such as BMP2, BMP7, BMP9 and OPN, decrease angiogenesis by suppressing HIF1 $\alpha$ and VEGF, and upregulate adipocyte transcription factor expression such as PPAR $\gamma 2$ and (442)aP2.
\end{abstract}

Keywords: Osteonecrosis; Mesenchymal Stromal Cells; Steroids; Bone Morphogenetic Proteins; Hypoxia-induciblefactor1 $\alpha$

\section{Introduction}

Osteonecrosis of femoral head (ONFH) is characterized by bone ischemia and microarchitectural deterioration, which lead a collapse of the femoral head during the late stage. ONFH represents a remarkable challenge to orthopedic surgeons and is a devastating disease for affected patients [1,2]. The use of corticosteroids such as dexamethasone and methylprednisolone for severe adult respiratory syndrome (SARS), rheumatoid diseases and organ transplantation has resulted in an increased risk of ONPH [3]. Intraosseous hypertension, thrombotic intravascular occlusion and extravascular compression by progressive accumulation of marrow fat stores are commonly accepted theories [4-6]. Although it is recognized that steroids administration and the development of steroids-induced ONFH are related, the precise pathogenesis remains largely unknown [7]. The pathobiological mechanism underlying the induction of adipogenesis and suppression of osteogenesis and angiogenesis by steroids has not been elucidated.

Human mesenchymal stromal cells (MSCs) are multipotent progenitors which undergo self-renewal and differentiate into osteogenic, angiogenic and adipogenic lineages [8]. It is suggested that ONFH is a disease of MSCs, due to abnormal proliferation or differentiation of MSCs [9]. Osteogenic differentiation is a sequential cascade that recapitulates most of the molecular events occurring during bone development and remodeling. Bone morphogenetic proteins (BMPs) play an important role during development and have been shown to regulate stem cell proliferation and osteogenic differentiation [10]. BMP2, 7 and 9 are the most potent BMPs among the 14 types of BMPs in inducing osteogenic differentiation of MSCs [11]. Normal and pathological bone physiology is inexorably tied to angiogenesis. The vasculature plays an important role for the mechanism of coupling resorption by osteoclasts and bone formation by osteoblasts [11]. Angiogenesis, the development of a microvascular network for blood supply, is critical for the development, remodeling and healing of bone [11-13]. Hypoxia can induce both apoptosis as well as necrosis of cells and is associated with vascular disease [12]. Hypoxia-inducible factor $1 \alpha$ (HIF1 $\alpha$ ) is a well established regulator of angiogenic cascade, which usually coordinates with skeletal development $[11,14]$. Vascular endothelial growth factor (VEGF), transcriptionally targeted gene of HIF1 $\alpha$, is a key regulator of vasculogenesis in the embryo and angiogenesis in adult tissues $[11,15,16]$. Thus, it appeared promising to investigate the role of angiogenic factors 
such as HIF1 $\alpha$ and VEGF. Small vessel occlusion by fatty emboli and the impedance of sinusoidal blood flow secondary to a rise in intraosseous pressure due to fatty infiltration following steroid therapy. In addition, steroid stimulates MSCs differentiation into adipocytes as well as the accumulation of fat in the marrow while suppressing cell differentiation into osteoblasts [17]. Peroxisome proliferator activated receptor $\gamma 2$ (PPAR $\gamma 2$ ) gene expression destines cells for adipocyte differentiation $[18,19]$.

Here, we investigate if the pathogenesis of steroid-induced osteonecrosis is associated with decreased proliferative capacity and abnormal differentiation of human MSCs at the proximal femur. The proliferation of cultured human MSCs in patients with corticosteroid-induced $\mathrm{ONFH}$ is depressed. We report the identification of abnormal differentiation in isolated MSCs and sections of whole femoral heads obtained during total hip replacement for glucocorticoid-induced osteonecrosis. The decreased expression of BMP2, BMP7, BMP9 and Osteopontin provide supportive evidence corticosteroid-induced inhibition of osteogenesis. Down-regulation of HIF $1 \alpha$, VEGF and VWF by glucocorticoids is directly responsible for disturbed angiogenesis. Over-expression of PPAR $\gamma 2$ and (442)aP2 suggests the corticosteroid-induced adipogenic differentiation. Taken together, our findings should not only expand our understanding of the molecular basis behind steroid-induced osteonecrosis, but also provide an opportunity to harness proliferative capacity and differentiation of MSCs in regenerative medicine.

\section{Materials and Methods}

\section{Patients}

The study was approved by the University Ethics Committee and informed consent was obtained from all patients. $12 \mathrm{ONFH}$ patients ( 4 men and 8 women, aged from 38 to 65 years old) with history of corticosteroid usage for rheumatoid arthritis, systemic lupus erythematosus and uveitis were recruited. All Patients with stage III and stage IV ONFH underwent total hip replacement (THR). The exclusion criteria included metabolic bone diseases, such as Paget's disease, renal osteodystrophy, hyper- or hypoparathyroidism and malignant tumors. The necrotic bone in the center of split femoral head was harvested for study. Bone specimens from additional 12 patients who underwent THR for femoral neck fractures were harvested and enrolled as controls. No age-matched control was obtained because of the absence of surgical indication for THR in young patients with femoral neck fractures.

\section{Harvest and identification of human mesenchymal stromal progenitor cells}

Bone marrow was obtained at the femoral neck and adherent stromal cells were harvest and cultured in the conditions as described [20]. Cells were treated with $0.1 \%$ triton-X for 10 minutes and blocked with phosphate-buffered saline containing $1 \%$ bovine serum albumin and mouse serum for 30 minutes. Fluorescein isothiocyanate-labeled mouse anti-human CD29 antibody (1:100, Santa Cruz) and PE-labeled rat antihuman CD44 (1:100, Santa Cruz) were added simultaneously to 1 well. Mouse antihuman CD34, or CD14 antibodies (Santa Cruz) were added; following incubation for 30 minutes, fluorescein isothiocyanate-labeled rabbit antimouse IgG antibody then was added. Identification of mesenchymal stem cells was performed as previously described [21].

\section{Measurement of proliferation of human mesenchymal stromal cells using Flow Cytometry}

When MSCs covered $90 \%$ of the flask, they were digested and resuspended at the density of $1 \times 10^{6} / \mathrm{mL}$. Cells were fixed in $80 \%$ pre-cooled alcohol at 4 overnight. After washing with PBS and centrifuged for 5 minutes, the supernatant was discarded. $10 \mu \mathrm{L}$ of RNase $(100 \mu \mathrm{g} / \mathrm{mL}), 10 \mathrm{~mL}$ of propidium iodide $(100$ $\mu \mathrm{g} / \mathrm{mL}$ ) and $40 \mu \mathrm{L}$ of PBS were added, and the cells were kept in darkness for 30 minutes. The cell cycles were measured with a flow cytometry instrument. The proliferation index (PI) was used to assess the levels of proliferation.

\section{RNA isolation and semi-quantitative RT-PCR}

Total RNA was isolated from subconfluent MSCs using TRIZOL Reagents (Invitrogen) and used to generate cDNA templates by RT reaction with hexamer and M-MuLV Reverse Transcriptase (New England Biolabs, Ipswich, MA). The first strand cDNA products were further diluted five to ten folds and used as PCR templates. Semiquantitative RT-PCR (sqPCR) was carried out as described [11]. PCR primers (supplementary material Table) were designed by using the Primer3 program to amplify the genes of interest (150-180 bp). A touchdown cycling program was as follows: $94^{\circ} \mathrm{C}$ for 2 minutes for 1 cycle; $92^{\circ} \mathrm{C}$ for 20 seconds, $68^{\circ} \mathrm{C}$ for 30 seconds, and $72^{\circ} \mathrm{C}$ for 12 cycles decreasing $1^{\circ} \mathrm{C}$ per cycle; and then at $92^{\circ} \mathrm{C}$ for 20 seconds, $57^{\circ} \mathrm{C}$ for 30 seconds, and $72^{\circ} \mathrm{C}$ for 20 seconds for 20-25 cycles, depending on the abundance of a given gene. PCR products were resolved on 1.5\% agarose gels. All samples were normalized by the expression level of GAPDH.

\section{Western blotting analysis}

Western blotting was carried out as previously described [11]. Briefly, tissues were collected in Lysis Buffer. Cleared total cell lysate was denatured by boiling and resolved by $10 \%$ SDS-PAGE. After electrophoretic separation, proteins were transferred to an Immobilon-P membrane. Membrane was blocked with SuperBlock Blocking Buffer, and probed with anti-BMP2 (Proteintech), BMP7 (Proteintech), BMP9 (Proteintech), Osteopontin (ab8448), HIF1 $\alpha$ (Proteintech), VEGF (Proteintech), PPAR 2 (Proteintech), vWF (Proteintech) and (442)aP2 (Proteintech) or anti-ß-actin (Santa Cruz), followed by incubation with a secondary antibody conjugated with horseradish peroxidase. The proteins of interest were detected by using SuperSignal West Pico Chemiluminescent Substrate kit.

\section{Immunohistochemical and Immunofluorescent staining}

Immunohistochemical staining on paraffin-embedded tissues was carried out with an anti-BMP2, BMP7, BMP9, HIF1 $\alpha$, VEGF, PPAR 2 , vWF and (442)aP2 antibody. The presence of the expected protein was visualized by DAB staining and examined under a microscope as previously described [11]. The slides were then incubated with primary antibody diluted in PBS containing $1 \%$ BSA for $1 \mathrm{~h}$. The primary antibodies used were as follows: 
anti-osteopontin, vWF and (442)aP2 antibody. After washing 3 times in PBS, AlexaFluor488, AlexaFluor555 (Invitrogen, Grand Island, NY) conjugated anti-rabbit or anti-mouse IgG was added in PBS with1\% BSA for $1 \mathrm{~h}$. After the final wash, 6-diamidino2-phenylindole (DAPI) (Sigma) was added and used as a counterstain for nuclei. Fluorescence images were acquired using an Olympus microscope with DP manager software.

\section{Test concentration of triglycerid reagent}

The tissues of the ONFH and control groups were lysed with NP40 buffer (1\% NP-40, $0.15 \mathrm{M} \mathrm{NaCl}, 50 \mathrm{mM}$ Tris, pH 8.0) containing protease inhibitors (Sigma). Protein quantitation was performed with BCA protein assay reagent (Pierce, Rockford, IL, USA). Equal amounts of protein from the different groups were added into the reaction of triglyceride reagent KIT for $5 \mathrm{~min}$ at 37 . The extracted dye was transferred to 96 -well plates, and the optical density was measured at $600 \mathrm{~nm}$.

\section{Statistical analysis}

All quantitative experiments were performed in triplicate and/or repeated three times. Data were expressed as mean \pm S.D. Statistical significances between vehicle treatments versus drugtreatment were determined by one-way analysis of variance and the Student's t-test. A value of $\mathrm{P}<0.05$ was considered statistically significant.

\section{Results}

\section{Clinical data of patients}

Representative radiograph and MRI showed femoral head erosion and collapse in the ONFH group and femoral neck fracture in the control group (Figure 1A and 1B). Bone marrow edema and femoral head necrosis were visible in the ONFH group (Figure 1C). This revealed significant accumulation of marrow fat stores of ONFH relative to the control of normal femoral head.

\section{Inhibited proliferation of MSCs in corticosteroid- induced osteonecrosis of femoral head}

We first identified the expression of typical MSCs markers. Immunofluorescent staining showed the cultured cells expressed typical MSCs markers such as CD29 and CD44 (Figure 2A and 2B), but not typical hematopoietic cell markers including CD34 and CD14 (data not shown). To investigate the potential role of steroid in affecting proliferative capability of MSCs, we examined growth period of MSCs between the ONFH and control groups using flow cytometry. The percentage of cells in the $\mathrm{G}_{2} / \mathrm{M}+\mathrm{S}$ stages is taken as the proliferation index (PI) to indicate the proliferation of cells. Compared with the control group, the percentage of cells in the $G_{0} / G_{1}$ stages in the ONFH group was increased significantly, whereas the percentage in $G_{2} / M+S$ stages (PI) was decreased significantly $(\mathrm{P}<0.01)$ (Figure $2 \mathrm{C}$ and $2 \mathrm{D})$.

Corticosteroid down-regulates the expression of BMP2, 7, 9 and late osteogenic marker in the ONFH and inhibits osteogenesis of MSCs in vivo

Bone morphogenetic proteins (BMPs) play an important role in regulating stem cell proliferation and osteogenic differentiation.

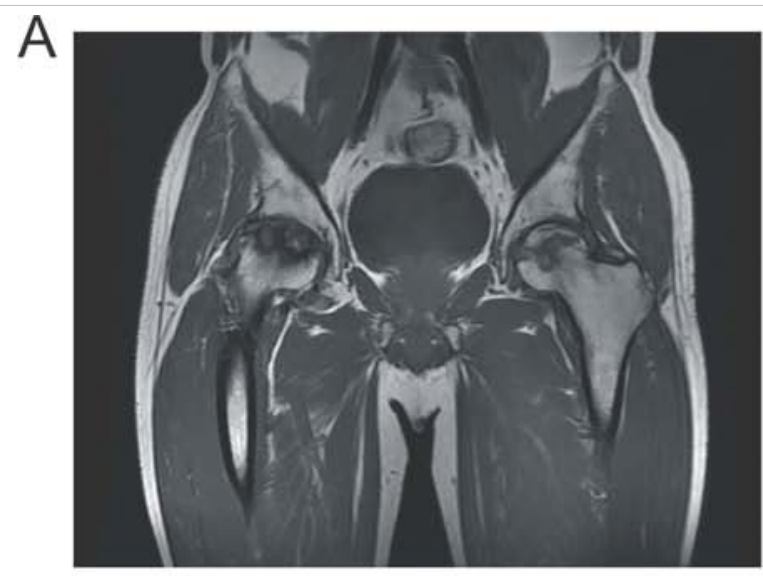

B
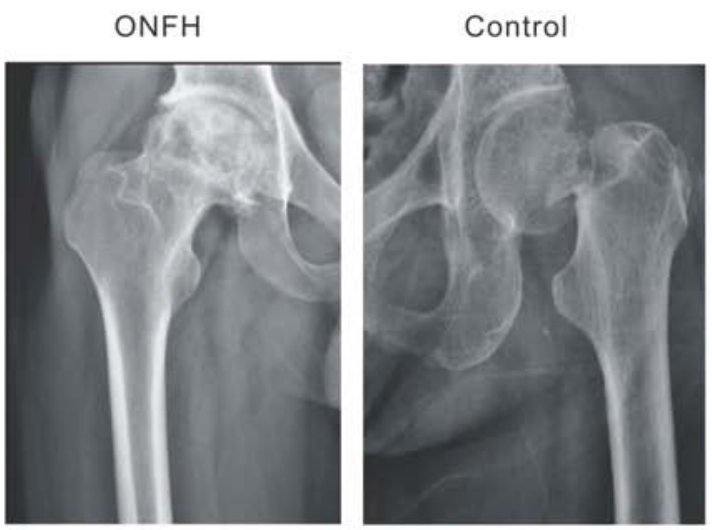

C

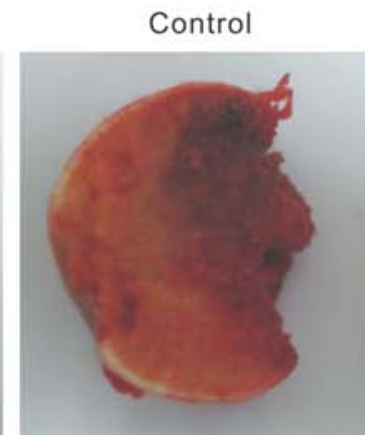

Figure 1: Clinical data of patients.

(A) MRI showed femoral head erosion and collapse in the ONFH group. (B) Representative radiograph showed femoral head necrosis in the ONFH group and femoral neck fracture in the control group.

(C) The split femoral heads were harvested for study. In the ONFH group, Bone marrow edema and femoral head necrosis were visible. In the control group, bone specimens from the cases who underwent THR for femoral neck fractures were harvested.

BMP2, 7 and 9 are the most potent BMPs among the 14 types of BMPs in inducing osteogenic differentiation of MSCs. Compared with those in the control group, we found the transcriptions of BMP2, 7 and 9 were significantly reduced by $93.8 \%, 64.3 \%$ and $96.4 \%$ respectively in the ONFH group (Figure 3A). Similarly, the protein expression levels of BMP2, 7 and 9 were significantly reduced in the ONFH group (Figure 3B). Immunohistochemical staining also confirmed that corticosteroid inhibited expression 

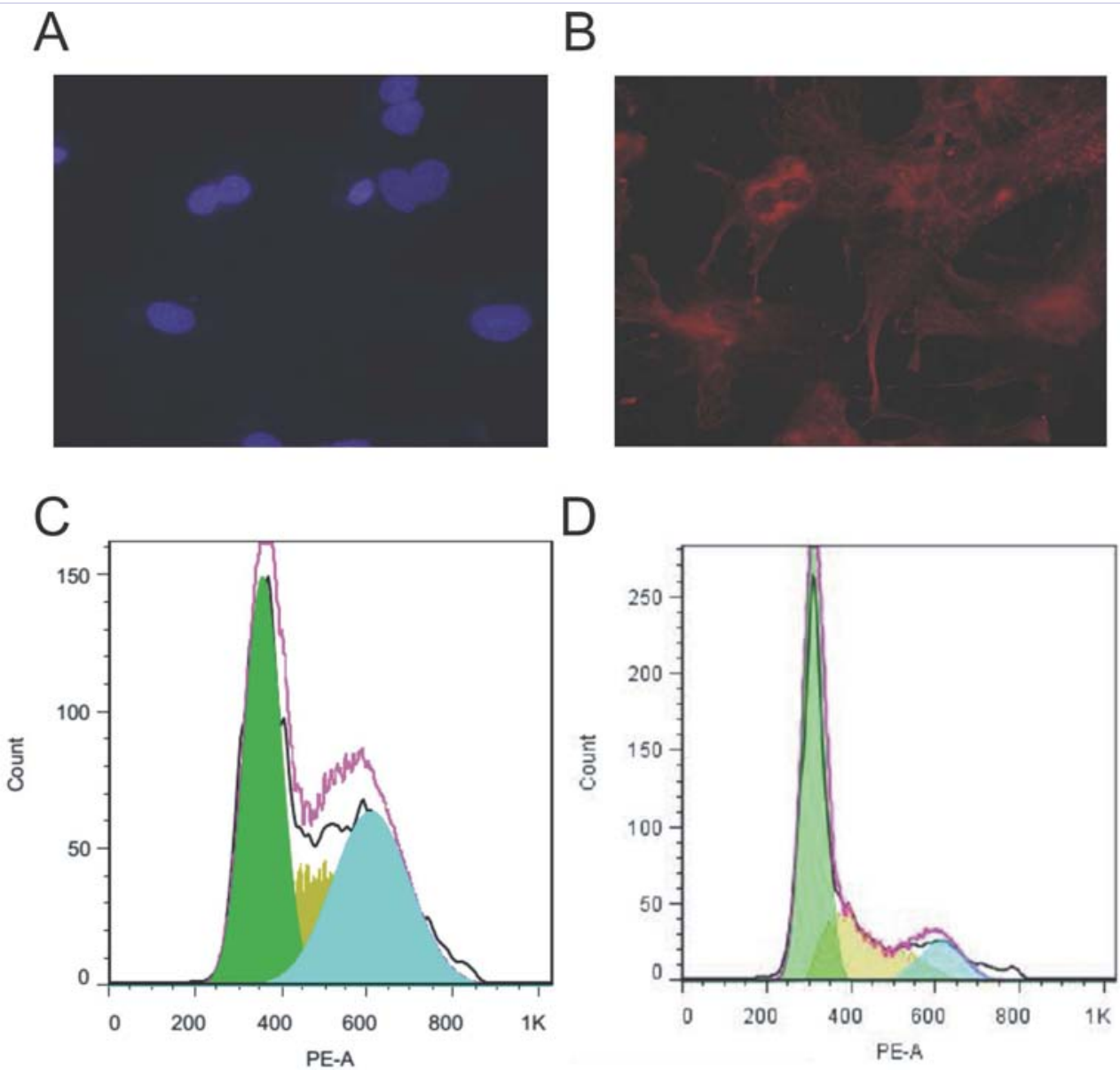

Figure 2: Decreased proliferation of MSCs in corticosteroid-induced ONFH.

The expression of the surface markers were determined by immunocytochemistry using the monoclonal antibodies CD29-FITC (A) and CD44-PE (B). Positive staining was observed in the cells $(\times 200)$. Growth period of flow cytometry in the control group (C) and the ONFH group (D). The percentage of cells in $\mathrm{G}_{2} / \mathrm{M}+\mathrm{S}$ stages was $59.54 \%$ and the percentage in the $\mathrm{G}_{0} / \mathrm{G}_{1}$ stages was $40.46 \%$ in the control group (C). The percentage in $\mathrm{G}_{2} / \mathrm{M}+\mathrm{S}$ stages was $37.45 \%$ and the percentage in the $G_{0} / G_{1}$ stages was $62.55 \%$ in the ONFH group (D).

of BMP2, 7 and 9 at protein levels (Figure 3C). We also found that corticosteroid was able to suppress late osteogenic marker osteopontin (OPN) at mRNA (Figure 3A) and protein (Figure 3B) levels. Immunofluorescent staining results also confirmed that osteopontin protein was significantly decreased in ONFH group relative to the control (Figure 3D).

Corticosteroid down-regulates the expression of HIF1, VEFG and vWF in the ONFH and inhibits angiogenesis of MSCs in vivo

HIF $1 \alpha$ is a well established regulator of angiogenic cascade. VEGF, transcriptionally targeted gene of $\mathrm{HIF} 1 \alpha$, is a key regulator of angiogenesis in adult tissues. And von Willebrand factor (vWF) is one of angiogenic markers. We found that mRNA of HIF1 $\alpha$, VEGF and vWF were inhibited by $98.2 \%, 62.3 \%$ and $69.93 \%$ respectively in the ONFH group, compared with those in the control group (Figure 4A). Western blot detected that protein expression levels of HIF1 $\alpha$, VEGF and vWF were inhibited significantly (Figure 4B). Immunohistochemical staining also confirmed that corticosteroid inhibited the expression of HIF1 $\alpha$,
VEGF and vWF at protein levels (Figure 4C). vWF protein expression level was also tested by Immunofluorescence and vWF was notably decreased in group of ONFH relative to the control(Figure 4D).

Corticosteroid upregulates the expression of PPAR $\gamma 2$, (442)aP2 and triglycerid in the ONFH and induced adipogenic differentiation of MSCs in vivo

The gene expression of PPAR $\gamma 2$ and (442)aP2 destine cells for adipocyte differentiation. We also found that mRNA level of PPAR 2 and (442)aP2 were augmented by 9.44 and 33.57 folds respectively in the ONFH group (Figure 5A). Protein expression of PPAR $\gamma 2$ and (442)aP2 were increased respectively (Figure 5B). Immunohistochemical staining also confirmed that corticosteroid augmented the expression of PPAR $\gamma 2$ and (442) aP2 at protein levels (Figure 5C). Immunofluorescent results displayed that (442)aP2 protein was significantly increased in $\mathrm{ONFH}$ group relative to the control(Figure 5D). In addition, triglycerid concentration was markedly increased in the $\mathrm{ONFH}$ (Figure 5E). 


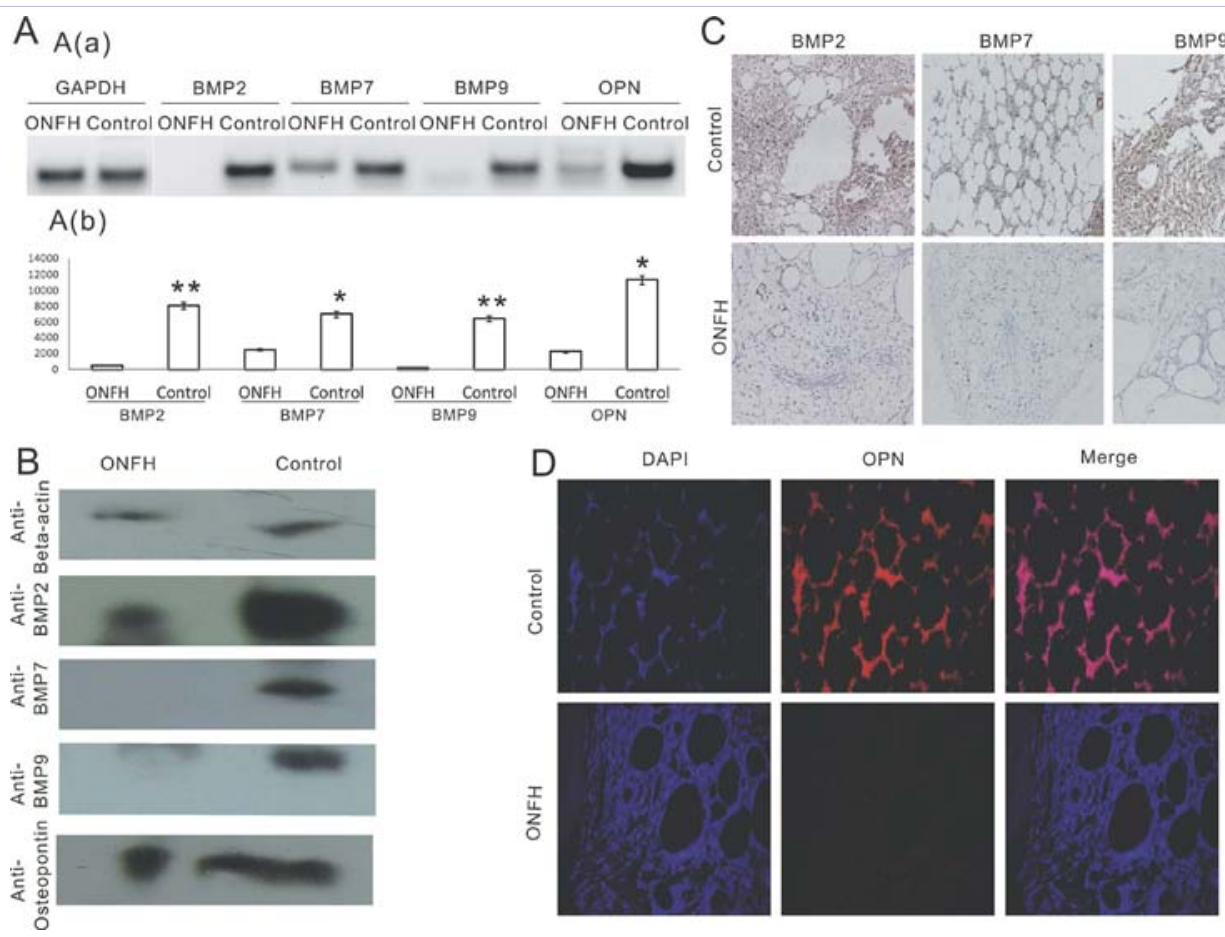

Figure 3: Corticosteroid down-regulates the expression of BMP2, 7, 9 and late osteogenic marker gene of osteopontin in the ONFH and inhibits osteogenesis of MSCs in vivo.

(A) The transcription expression of BMP2, BMP7, BMP9 and OPN in MSCs of the ONFH group and the control group. Total RNA was isolated from subconfluent cells and subjected to semi-quantitative PCR (sqPCR) analysis using primers specific for human BMP2, BMP7, BMP9 and OPN. Expected PCR products were resolved on agarose gels (a). The signal intensities of the expected products were quantitatively analyzed using the NIH ImageJ software (b). Each PCR condition was done in triplicate. Representative results are shown.

(B) The protein expression of BMP2, BMP7, BMP9 and OPN in MSCs of the ONFH group and the control group. Tissues were prepared in the same fashion as described in (a). Tissues were lyzed and subjected to SDS-PAGE and Western blotting with anti-BMP2, anti-BMP7, anti-BMP9 and anti-OPN antibodies. Expression levels of $\beta$-actin was used to assess equal loading of total lysate.

(C) Immunohistochemical staining of BMP2, BMP7 and BMP9 in the sections of the ONFH and control groups. Bone marrow was retrieved in a similar fashion as shown in Figure 1C. The retrieved tissues were subjected to BMP2, BMP7 or BMP9 antibody immunohistochemical staining. Isotype IgG was used as a negative control (not shown). Representative results are shown. Magnification, 40x.

(D) Immunofluorescent staining results also confirmed that osteopontin protein was significantly decreased in ONFH group relative to the control. Each assay condition was done in triplicate. ${ }^{*} p<0.05 ; * * 0.001$.

Table 1: PCR Oligonucleotides.

\begin{tabular}{|l|l|}
\hline human BMP2 Fwd: & ACTCGAAATTCCCCGTGACC \\
\hline human BMP2 Rev: & CCACTTCCACCACGAATCCA \\
\hline human BMP7 Fwd: & GACTTCAGCCTGGACAACGA \\
\hline human BMP7 Rev: & TGTAGGGGTAGGAGAAGCCC \\
\hline human BMP9 Fwd: & CTGTGGAGAGCCACAGGAAG \\
\hline human BMP9 Rev: & CTCCTTTTCCGCCTGGCTAA \\
\hline human OPN Fwd: & CATACAAGGCCATCCCCGTT \\
\hline human OPN Rev: & TGGGTTTCAGCACTCTGGTC \\
\hline human HIF1 $\alpha$ Fwd: & TGCATCTCCATCTCCTACCC \\
\hline human HIF1 $\alpha$ Rev: & CGTTAGGGCTTCTTGGATGA \\
\hline human VEGF Fwd: & CCCACTGAGGAGTCCAACAT \\
\hline human VEGF Rev: & TTTCTTGCGCTTTCGTTTTT \\
\hline human vWF Fwd: & CCACTTGCCACAACAACATC \\
\hline human vWF Rev: & TGGACTCACAGGAGCAAGTG \\
\hline human PPAR $\gamma 2$ Fwd: & ATCTTTCAGGGCTGCCAGT \\
\hline
\end{tabular}

\begin{tabular}{|l|l|}
\hline human PPAR 2 Rev: & GGAGGCCAGCATTGTGTAA \\
\hline human (442)aP2 Fwd: & GGTGCAGAAGTGGGATGG \\
\hline human (442)aP2 Rev: & TGGCTCATGCCCTTTCAT \\
\hline human GAPDH Fwd: & GCATGGCCTTCCGTGTCCCC \\
\hline human GAPDH Rev: & GAGGGCAATGCCAGCCCCAG \\
\hline
\end{tabular}

\section{Discussion}

Corticosteroid has been the focus of studies on the pathogenesis of ONFH. Patients receiving steroid therapy have an approximately 20 -fold increase in their likelihood of developing ONFH [22]. Though the dose effect of corticosteroid therapy on osteonecrosis remains largely unknown, recent studies suggest that corticosteroid doses above $25-40 \mathrm{mg} /$ day are significant risk factors for nontraumatic ON in renal transplant and SLE patients [22]. The number of MSCs at the proximal femoral was significantly decreased in the corticosteroid-induced osteonecrosis compared to other kinds of osteonecrosis [23]. Corticosteroid can promote apoptosis of osteoblasts and

Citation: Wang C, Huang W, Gong X, Ding G, Liang X, et al. (2014) Decreased Proliferation and Abnormal Differentiation of Human Mesenchymal Stromal Cells in Steroid-Induced Osteonecrosis of Femoral Head. SOJ Biochem 1(1), 8. DOI: http://dx.doi. 

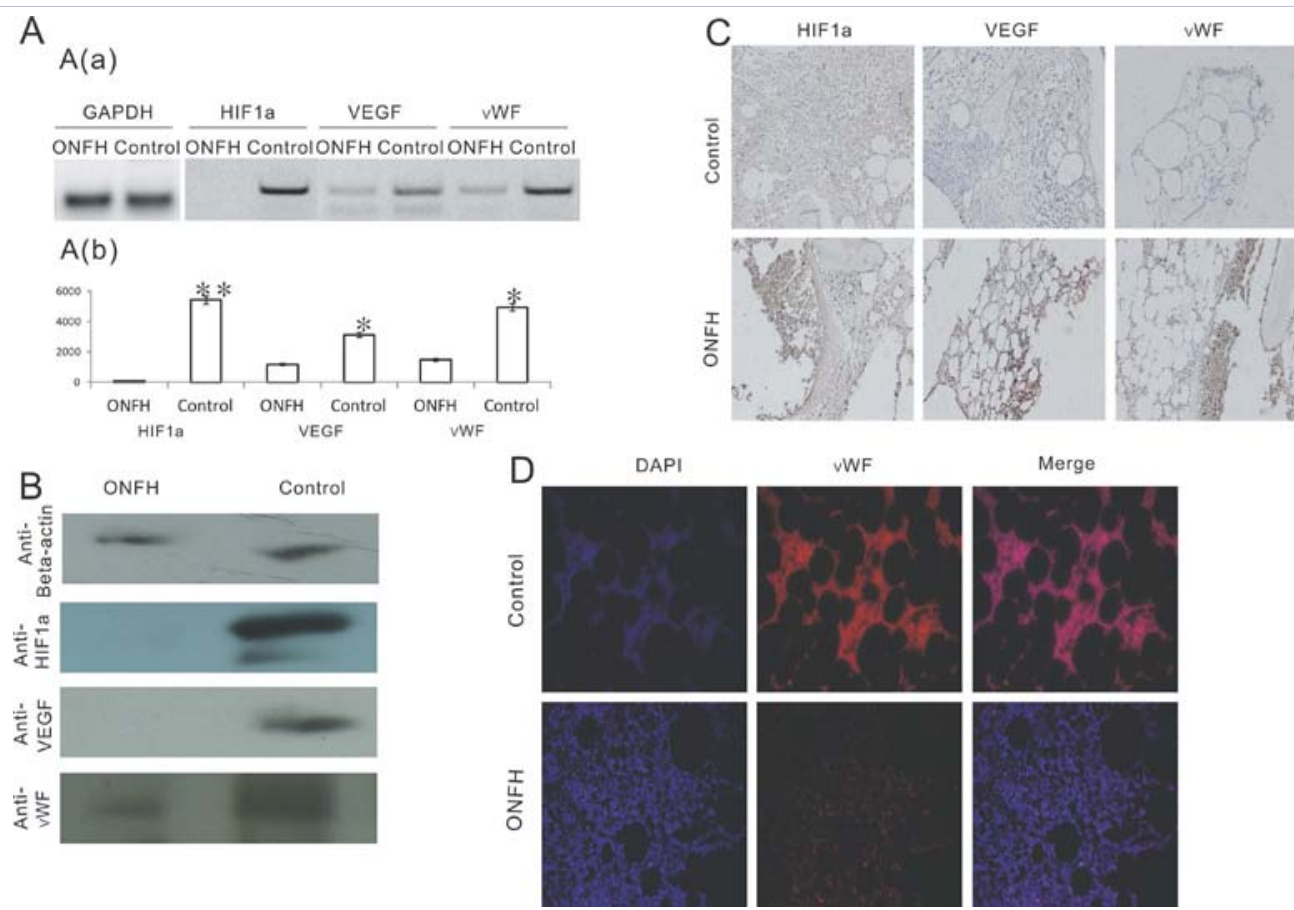

Figure 4: Corticosteroid down-regulates the expression of HIF1a, VEFG and vWF in the ONFH and inhibits angiogenesis of MSCs in vivo.

(A) The transcription expression of HIF1a, VEGF and vWF in MSCs of the ONFH group and the control group. Total RNA was isolated from subconfluent cells and subjected to semi-quantitative PCR (sqPCR) analysis using primers specific for human HIF1a, VEGF and vWF. Expected PCR products were resolved on agarose gels (a). The signal intensities of the expected products were quantitatively analyzed using the NIH ImageJ software (b). Each PCR condition was done in triplicate. Representative results are shown.

(B) The protein expression of HIF1a, VEGF and vWF in MSCs of the ONFH group and the control group. Tissues were prepared in the same fashion as described in (a). Tissues were lyzed and subjected to SDS-PAGE and Western blotting with anti-HIF1a, anti-VEGF and anti-vWF antibodies. Expression levels of $\beta$-actin was used to assess equal loading of total cell lysate.

(C) Immunohistochemical staining of HIF1a, VEGF and vWF in the sections of the ONFH and control groups. Bone marrow was retrieved in a similar fashion as shown in Figure 1. The retrieved tissues were subjected to HIF1a, VEGF or vWF antibody immunohistochemical staining. Isotype IgG was used as a negative control (not shown). Representative results are shown. Magnification, 40x.

(D) vWF protein expression level was also tested by Immunofluorescence and vWF was notably decreased in group of ONFH relative to the control. Each assay condition was done in triplicate. ${ }^{*} p<0.05$; $^{* *} p<0.001$.

osteocytes and suppress the proliferative capacity of MSCs, leading to a decrease in osteocytes [24-26]. Our results also confirmed that corticosteroid induces osteonecrosis through lowering the proliferative activity or differential capacity as well as altering the differentiation direction of MSCs.

Osteogenic differentiation is a sequential cascade that recapitulates most of the molecular events occurring during embryonic skeletal development [10]. BMPs belong to the transforming growth factor $\beta$ (TGF $\beta$ ) superfamily and consist of at least 14 members in humans [8]. Genetic disruptions of BMPs have resulted in various skeletal and extraskeletal abnormalities during development [11]. BMP2, 7 and 9 are the most potent BMPs in inducing osteogenic differentiation of MSCs by regulating several important downstream targets during BMPs-induced osteoblast differentiation [8,11]. Our results confirmed that corticosteroid down-regulates the expression of BMP2, 7 and 9, thus reduces late osteogenic markers and inhibits osteogenesis of MSCs.

The process of bone development and repair depends on adequate formation of new capillaries from existing blood vessel
$[11,12]$. It has been reported that angiogenesis and osteogenesis are well coordinated processes during bone development [11]. We have demonstrated that BMP9 directly upregulates HIF1 $\alpha$ expression in MSCs, which in turn induces both osteogenic factors and angiogenic factor VEGF [11]. Thus, potent osteogenic factors, such as BMP9, may induce a tightly-regulated convergence of osteogenic and angiogenic signaling in MSCs, and subsequently lead to efficient bone formation. HIF1 regulates target genes such as VEGF and vWF that mediate adaptive responses, such as angiogenesis, to reduced oxygen availability [11]. HIF1 $\alpha$ polymorphisms are associated with idiopathic ONFH in men; and variations in HIF1 $\alpha$ play a role in the pathogenesis and risk factor for ONFH [27]. Osteoblasts derived from femoral heads have been found to exhibit downregulation of VEGF within 24 hours of incubation with corticosteroid [28]. Down-regulation of HIF1 $\alpha$, VEGF and vWF by corticosteroid is directly responsible for disturbed angiogenesis resulting in the defects in capillary architecture, which eventually lead to osteonecrosis.

Corticosteroid can induce differentiation of MSCs into adipose cells. Since adipocytes and osteoblasts share a common progenitor pool, when exogenous stimulators shift the differentiation of 

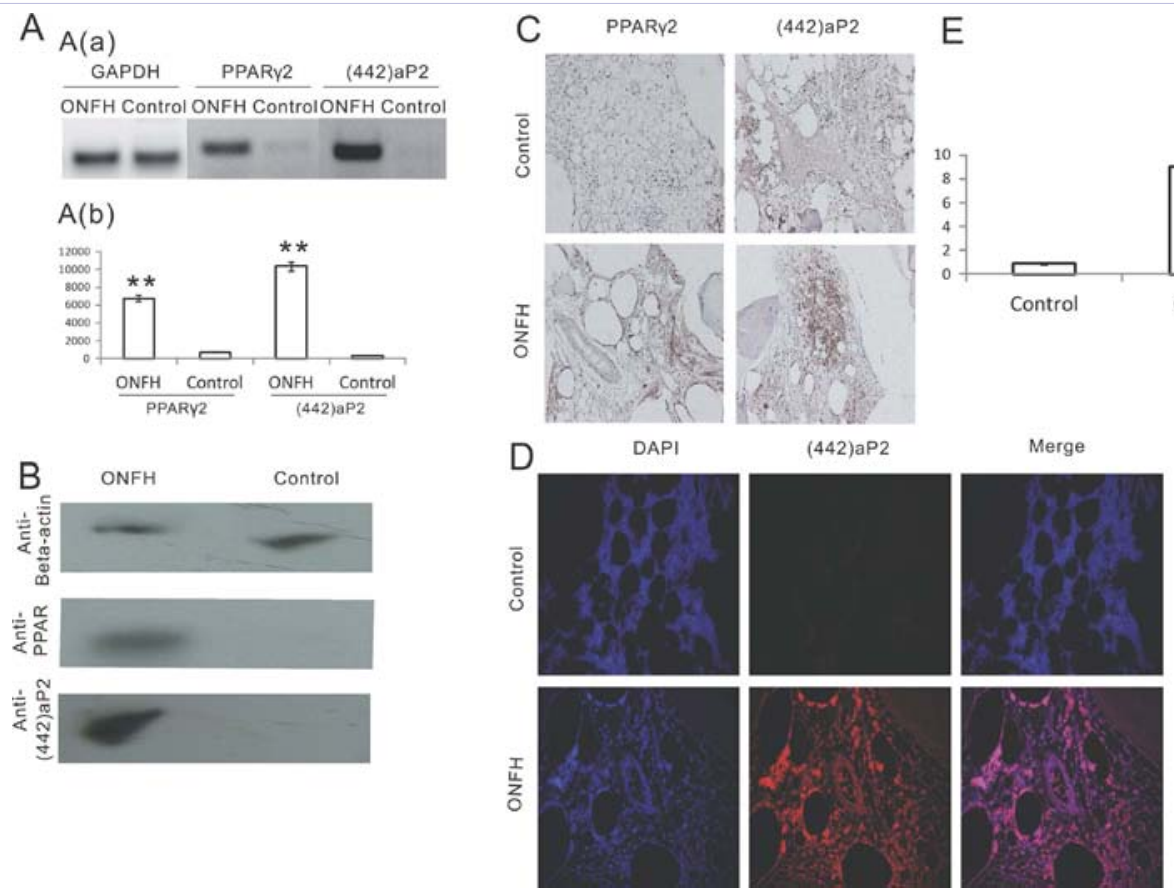

Figure 5: Corticosteroid upregulates the expression of PPAR $\gamma 2$, (442)aP2 and triglycerid in the ONFH and induced adipogenic differentiation of MSCs in vivo.

(A) The transcription expression of PPAR $\gamma 2$ and (442)aP2 in MSCs of the ONFH group and the control group. Total RNA was isolated from subconfluent cells and subjected to semi-quantitative PCR (sqPCR) analysis using primers specific for human PPAR 2 and (442)aP2. Expected PCR products were resolved on agarose gels $(a)$. The signal intensities of the expected products were quantitatively analyzed using the NIH ImageJ software $(b)$. Each PCR condition was done in triplicate. Representative results are shown.

(B) The protein expression of PPAR 2 and (442)aP2 in MSCs of the ONFH group and the control group. Tissues were prepared in the same fashion as described in (a). Tissues were lyzed and subjected to SDS-PAGE and Western blotting with anti- PPAR 2 and anti-(442)aP2 antibodies. Expression levels of $\beta$-actin was used to assess equal loading of total cell lysate.

(C) Immunohistochemical staining of PPAR $\gamma 2$ and (442)aP2 in the sections of the ONFH and control groups. Bone marrow was retrieved in a similar fashion as shown in Figure 1. The retrieved tissues were subjected to PPAR 2 or (442)aP2 antibody immunohistochemical staining. Isotype IgG was used as a negative control (not shown). Representative results are shown. Magnification, 40x.

(D) Immunofluorescent results displayed that (442)aP2 protein was significantly increased in ONFH group relative to the control.

(E) Detection of the concentration of triglyceride in bone tissues by TG reagent kit. The TG concentration of the control group was $0.87 \pm 0.03 \mathrm{mmol} / \mathrm{l}$ and the concentration of the $\mathrm{ONFH}$ group was $9.07 \pm 0.28 \mathrm{mmol} / \mathrm{L}$. Each assay condition was done in triplicate. ${ }^{*} p<0.05 ;{ }^{* *} p<0.001$.

MSCs into the adipocyte lineage, the osteoprogenitor pool is not sufficient to provide enough osteoblasts in order to meet the need for bone remodeling and repair of ONFH [29]. Fat cell hypertrophy has been observed in histologic specimens of human femoral heads following treatment with dexamethasone for 5 days [30]. Dexamethasone affects osteoblasts by inhibit the expression of type-I collagen and osteocalcin, thereby inducing osteoblast and osteocyte apopotosis [31-33]. It also down-regulates the expression of Cbfa1/Runx2 and osteocalcin promoter activity while it increases the expression of PPARc2 [29, 34].

\section{Conclusion}

There may be several mechanisms that are involved in the pathogenesis of osteonecrosis. Corticosteroid reduces the proliferative activity of MSCs. It down-regulates osteoblast transcription factor gene expression such as BMPs, decreases angiogenesis by suppressing HIF1 $\alpha$ and VEGF, and up-regulates adipocyte transcription factor expression. Consequently, the action impairs the differentiation of MSCs and decreases blood supply, leading to bone cell death.

\section{Acknowledgement}

The reported work was supported by research grants from Chongqing Science and Technology Commission foundation base and frontier research project (cstc2013jcyjA10033 to N. $\mathrm{Hu}, \mathrm{cstc2014jcyjA10024}$ to Wang) and the project of Chongqing Health Bureau (2013-2-005 to N. Hu), the doctoral funding of Ministry of Education of China (20125503120015 to C. Wang) and funding of Chongqing Medical University (0800280031 to C. Wang), and the Natural Sciences Foundation of China (\#81391972 to W. Huang). The funders had no role in study design, data collection and analysis, decision to publish, or preparation of the manuscript.

\section{References}

1. Mont MA, Jones LC, Hungerford DS. Nontraumatic osteonecrosis of the femoral head: ten years later. J Bone Joint Surg Am. 2006; 88(5):11171132.

2. Mont MA, Marulanda GA, Jones LC, Saleh KJ, Gordon N, Hungerford DS, et al. Systematic analysis of classification systems for osteonecrosis of 
the femoral head. J Bone Joint Surg Am. 2006; 88 Suppl 3:16-26.

3. Bradbury G, Benjamin J, Thompson J, Klees E, Copeland J. Avascular necrosis of bone after cardiac transplantation. Prevalence and relationship to administration and dosage of steroids. J Bone Joint Surg Am 1994; 76(9):1385-1388.

4. Hungerford DS, Lennox DW. The importance of increased intraosseous pressure in the development of osteonecrosis of the femoral head: implications for treatment. Orthop Clin North Am. 1985: 16(4):635654.

5. Jones LC, Mont MA, Le TB, Petri M, Hungerford DS, Wang P, et al. Procoagulants and osteonecrosis. J Rheumatol. 2003; 30(4):783-791.

6. Wang GJ. The Frank Stinchfield Award paper. Improvement of femoral head blood flow in steroid-treated rabbits using lipid-clearing agent Hip. 1987; 87-93.

7. Wang GJ, Cui Q, Balian G. The Nicolas Andry award. The pathogenesis and prevention of steroid-induced osteonecrosis. Clin Orthop Relat Res. 2000; 370:295-310.

8. Deng ZL, Sharff KA, Tang N, Song WX, Luo J, Luo X, et al. Regulation of osteogenic differentiation during skeletal development. Front Biosci. 2008; 13:2001-2021.

9. Gangji V, Hauzeur JP, Schoutens A, Hinsenkamp M, Appelboom T, Egrise D. Abnormalities in the replicative capacity of osteoblastic cells in the proximal femur of patients with osteonecrosis of the femoral head. J Rheumatol. 2003; 30(2):348-351

10. Olsen BR, Reginato AM, Wang W. Bone development. Annu Rev Cell Dev Biol. 2000; 16:191-220.

11. Hu N, Jiang D, Huang E, Liu X, Li R, Liang X, et al. BMP9-regulated angiogenic signaling plays an important role in the osteogenic differentiation of mesenchymal progenitor cells. J Cell Sci. 2013; 126(Pt 2):532-41. doi: 10.1242/jcs.114231.

12. Streeten EA, Brandi ML. Biology of bone endothelial cells. Bone Miner. 1990; 10(2):85-94.

13. Colnot C, Thompson Z, Miclau T, Werb Z, Helms JA. Altered fracture repair in the absence of MMP9. Development. 2003; 130(17):41234133.

14. Majmundar AJ, Wong WJ, Simon MC. Hypoxia-inducible factors and the response to hypoxic stress. Mol Cell. 2010; 40(2):294-309.

15. Tatsuyama K, Maezawa Y, Baba H, Imamura Y, Fukuda M. Expression of various growth factors for cell proliferation and cytodifferentiation during fracture repair of bone. Eur J Histochem. 2000; 44(3):269-278.

16. Komatsu DE, Hadjiargyrou M. Activation of the transcription factor HIF-1 and its target genes, VEGF, HO-1, iNOS, during fracture repair Bone. 2004; 34(4): 680-688.

17. Cui Q, Wang GJ, Balian G. Steroid-induced adipogenesis in a pluripotential cell line from bone marrow. J Bone Joint Surg Am. 1997; 79(7):1054-1063.

18. Mueller E, Drori S, Aiyer A, Yie J, Sarraf P, Chen H, et al. Genetic analysis of adipogenesis through peroxisome proliferator-activated receptor gamma isoforms. J Biol Chem. 2002; 277(44):41925-41930.

19. Shao D, Lazar MA. Peroxisome proliferator activated receptor gamma, CCAAT/enhancer-binding protein alpha, and cell cycle status regulate the commitment to adipocyte differentiation. J Biol Chem. 1997; 272(34):21473-21478.

20.Wang BL, Sun W, Shi ZC, Lou JN, Zhang NF, Shi SH, et al. Decreased proliferation of mesenchymal stem cells in corticosteroid-induced osteonecrosis of femoral head. Orthopedics. 2008; 31(5):444.

21.Suh KT, Kim SW, Roh HL, Youn MS, Jung JS. Decreased osteogenic differentiation of mesenchymal stem cells in alcohol-induced osteonecrosis. Clin Orthop Relat Res. 2005; (431):220-225.

22. Sakaguchi M, Tanaka T, Fukushima W, Kubo T, Hirota Y. Impact of oral corticosteroid use for idiopathic osteonecrosis of the femoral head: a nationwide multicenter case-control study in Japan. J Orthop Sci. 2010; 15(2):185-191. doi: 10.1007/s00776-009-1439-3.

23. Hernigou P, Beaujean F, Lambotte JC. Decrease in the mesenchymal stem-cell pool in the proximal femur in corticosteroid-induced osteonecrosis. J Bone Joint Surg Br. 1999; 81(2):349-355.

24. Weinstein RS, Nicholas RW, Manolagas SC. Apoptosis of osteocytes in glucocorticoid-induced osteonecrosis of the hip. J Clin Endocrinol Metab. 2000; 85(8):2907-2912.

25. Lee JS, Lee JS, Roh HL, Kim CH, Jung JS, Suh KT. Alterations in the differentiation ability of mesenchymal stem cells in patients with nontraumatic osteonecrosis of the femoral head: comparative analysis according to the risk factor. J Orthop Res. 2006; 24(4):604-609.

26. Yin L, Li YB, Wang YS. Dexamethasone-induced adipogenesis in primary marrow stromal cell cultures: mechanism of steroid-induced osteonecrosis. Chin Med J (Engl). 2006; 119(7):581-588.

27. Hong JM, Kim TH, Chae SC, Koo KH, Lee YJ, Park EK, et al. Association study of hypoxia inducible factor 1alpha (HIF1alpha) with osteonecrosis of femoral head in a Korean population. Osteoarthritis Cartilage. 2007; 15(6):688-694.

28. Varoga D, Drescher W, Pufe M, Groth G, Pufe T. Differential expression of vascular endothelial growth factor in glucocorticoid-related osteonecrosis of the femoral head. Clin Orthop Relat Res. 2009; 467(12):3273-3282. doi: 10.1007/s11999-009-1076-3.

29. Li X, Jin L, Cui Q, Wang GJ, Balian G. Steroid effects on osteogenesis through mesenchymal cell gene expression. Osteoporos Int. 2005;16(1):101-108.

30. Kitajima M, Shigematsu M, Ogawa K, Sugihara H, Hotokebuchi T. Effects of glucocorticoid on adipocyte size in human bone marrow. Med Mol Morphol. 2007; 40(3):150-156.

31. Canalis E, Delany AM. Mechanisms of glucocorticoid action in bone. Ann N Y Acad Sci. 2002; 966:73-81.

32.0'Brien CA, Jia D, Plotkin LI, Bellido T, Powers CC, Stewart SA, et al. Glucocorticoids act directly on osteoblasts and osteocytes to induce their apoptosis and reduce bone formation and strength. Endocrinology. 2004; 145(4):1835-1841.

33. Maes C, Carmeliet P, Moermans K, Stockmans I, Smets N, Collen D, et al. Impaired angiogenesis and endochondral bone formation in mice lacking the vascular endothelial growth factor isoforms VEGF164 and VEGF188. Mech Dev. 2002; 111(1-2):61-73.

34. Weinstein RS, Jia D, Powers CC, Stewart SA, Jilka RL, Parfitt AM Manolagas SC. The skeletal effects of glucocorticoid excess override those of orchidectomy in mice. Endocrinology. 2004; 145(4):19801987. 